\title{
ORTEGA Y KUHN: FILOSOFÍAS PARALELAS EN BASE A SUS FUENTES
}

\section{ORTEGA AND KUHN: PARALLEL PHILOSOPHIES ON THEIR SOURCES}

\author{
Rodolfo Gutiérrez Simón ${ }^{1}$ \\ Universidad Complutense de Madrid
}

Recibido: 16-7-2015

Aceptado: 5-3-2015

\begin{abstract}
Resumen: El presente estudio trata de mostrar los profundos paralelismos que existen entre las obras de Ortega y las de Kuhn, con especial énfasis en el ámbito de la historia y la historia de la ciencia. Además, se propondrán hipótesis en torno a la causa de dichos paralelismos, atendiendo sobre todo a la lectura común de William James y Max Weber; y a la influencia indirecta de Alexander Bain.
\end{abstract}

Palabras clave: Ortega y Gasset; Kuhn; William James; creencias; historia de la ciencia.

\begin{abstract}
The aim of this paper is to show the similarity in Ortega's and Kuhn's works, specially related to History and History of Science. In addition, some hypotheses will be proposed on the cause for that similarity, focusing on the influence from William James and Max Weber, and the indirect influence from Alexander Bain too.
\end{abstract}

Key words: Ortega y Gasset; Kuhn; William James; beliefs; history of science.

1. (rodolfo.gutierrez@ucm.es) Doctorando en la Universidad Complutense de Madrid (España), Facultad de Filosofía, bajo la dirección de José Luis Villacañas Berlanga y Antonio Rivera García, con la tesis Ortega y el pragmatismo norteamericano. Influencias y confluencias. Contrato predoctoral de personal investigador en formación en la Universidad Complutense de Madrid (Departamento de Historia de la Filosofía, Estética y Teoría del Conocimiento). Miembro del Consejo de Redacción de la Revista Hispanismo Filosófico, componente del GIPEL y colaborador de la Biblioteca Saavedra Fajardo de Pensamiento Político Hispánico. Líneas de investigación: la filosofía de Ortega y Gasset; pragmatismo norteamericano y europeo; filosofía española e iberoamericana; historia de la filosofía. Algunas publicaciones recientes: "Los límites del sujeto y la libertad en Ortega y Gasset", Daimon. Revista internacional de filosofía, $\mathrm{n}^{\circ}$ 69, 2016, pp. 109-123; "Sobre la interpretación del concepto orteguiano de 'vocación"', Revista de Estudios Orteguianos, n 31, 2015, pp. 115-139; "México en la mirada anglosajona: buscando al Humboldt integral", Res Publica. Revista de Historia de las Ideas Políticas, vol. 19, nº 1 (2016), pp. 243-250. 
¡De tal modo son los siglos como climas favorables o adversos a determinadas simientes ideológicas!

J. Ortega y Gasset, Introducción a una estimativa. ¿Qué son los valores?

\section{Introducción}

Aunque considero que el pensamiento de José Ortega y Gasset tiene validez por sí mismo, es indudable que en el ámbito de la historia de la ciencia no le ha sido concedida la relevancia que pudo merecer. Tal preponderancia sí se le ha otorgado a otro pensador de reconocido prestigio y especializado estrictamente en dicha disciplina, Thomas Samuel Kuhn. A la luz de ello, me propongo mostrar en este artículo no sólo la clara semejanza entre la postura de ambos autores en lo referente a la historia de la ciencia como disciplina (aspecto fundamental de este texto), sino también dos tareas subsidiarias: mostrar el paralelismo que se produce entre ambas filosofías en otros ámbitos y sugerir posibles influencias comunes de raigambre pragmatista en su mayor parte. Con ello espero conseguir no sólo que se preste la atención debida a Ortega en el campo de la filosofía y la metodología de la ciencia, sino también allanar el camino a la hora de abrir nuevas líneas de investigación respecto a la filosofía del autor madrileño que presten atención a las fuentes no germánicas de su pensamiento.

\section{Algunas aclaraciones iniciales}

Pese a que el objetivo de este artículo es mostrar las claras convergencias entre la obra kuhniana y la orteguiana, es preciso antes explicitar que existen entre ambas diferencias muy evidentes. En primer lugar, es preciso señalar que Ortega y Kuhn se mueven en ámbitos biográficos y filosóficos distintos, siendo también diferentes sus objetivos. Así, el pensador estadounidense concentrará sus esfuerzos en la reflexión en torno a la ciencia, mientras que la filosofía de Ortega abarca todos los ámbitos del conocimiento y la vida humana. Visto desde la perspectiva del autor madrileño, esto supone una ventaja y una desventaja. El pro radica en 
que, siendo un pensador sistemático ${ }^{2}$, aquellos puntos de su concepción en torno a la ciencia que no estén del todo claros pueden explicarse recurriendo a su pensamiento en torno al arte, la política u otros elementos; el contra, por su parte, es que esta amplitud de temas implica una menor profundización en los detalles. Así las cosas, Ortega sólo algunas veces (especialmente, y de manera muy peculiar, en su libro En torno a Gali$l e o^{3}$, que inicialmente iba a llevar el más acertado título Esquema de las crisis) se referirá estrictamente a la historia de la ciencia, siendo ésta un caso particular en el ámbito más amplio de la historia en general ${ }^{4}$. A este respecto, puedo ya adelantar que el objetivo de dicho texto -En torno a Galileo- es analizar las creencias imperantes en la época del genial autor italiano, a qué creencias sustituían, etc.; es decir, exactamente el mismo propósito que sobre la época de Copérnico se propone explícitamente Kuhn en La revolución copernicana ${ }^{5}$. Sea como fuere, y volviendo a Ortega, en «Guillermo Dilthey y la idea de la vida» ${ }^{6}$ se ve cómo el autor tiene una altitud de miras que rebasa la historia de la ciencia, aunque la incluye: la relaciona con la filosofía de la historia, formando parejas de una y otra disciplina. Tales parejas se irían sucediendo en el tiempo, puliendo lo que las anteriores dejaron tan alto como pudieron: Copérnico-Vico, Brahe-Boyle, Kepler-Voltaire, Galileo-Montesquieu...

Igualmente, me gustaría advertir que Kuhn trata de explicar cómo funciona efectivamente la ciencia; Ortega, en cambio, se sitúa en un plano no sólo descriptivo sino también normativo: su intención, en unas circunstancias como las de la España de la primera mitad del siglo XX, es partir de la situación real y modificarla; esto es, pretende que la situación deje de ser la que es (la que era) para convertirse en la que, a su juicio, debería ser. Baste en este sentido leer El tema de nuestro tiempo: cuando diferencia

2. Sobre la sistematicidad o no del pensamiento orteguiano se ha debatido en multitud de ocasiones. La mejor muestra de tal sistematicidad la podemos encontrar en «Estética en el tranvía», cuando afirma: «Como en belleza, así en ética» (Ortega y Gasset, J.: Obras Completas, Madrid: Taurus, 2004-2010, 10 vols., Tomo II, p. 181. En adelante, citaré a Ortega por estas obras completas como $O C$, indicando a continuación el tomo en romanos y la página en arábigos).

3. Puede leerse en $O C$ VI, 367-506. Naturalmente, estoy exagerando: hay más lugares en los que Ortega se refiere explícitamente a la cuestión, como por ejemplo «Vicisitudes de las ciencias», OC, IV, 292 ss.

4. La historia es un tema decisivo en su reflexión. Si hubiera que restringirse a un único texto que, de manera esquemática, englobe los puntos cruciales de su doctrina al respecto, habrá que remitirse a Historia como sistema, OC VI, 45-81.

5. Kuhn, T. S.: La revolución copernicana. La astronomía planetaria en el desarrollo del pensamiento, Barcelona: Ariel, 1996, pp. 27-28.

6. Concretamente, cf. OC VI, 234.

Thémata. Revista de Filosofía N54 (2016) pp.: 53-72. 
entre innovadores y conservadores en ciencia $^{7}$ se aprecia nítidamente que se considera a sí mismo como un innovador ${ }^{8}$.

Entre Kuhn y Ortega hay también otras diferencias. Aunque advertirlas no es el objetivo de este texto (todo lo contrario), me gustaría explicitar una, a fin de dotar a mi argumentación de fidelidad a las fuentes. Recurriré para ello al artículo de Ortega «Las dos grandes metáforas (En el segundo centenario del nacimiento de Kant) $»^{9}$. Podemos ahí leer lo siguiente:

Dos usos de rango diferente tiene en la ciencia la metáfora. Cuando el investigador descubre un fenómeno nuevo, es decir, cuando forma un nuevo concepto, necesita darle un nombre. Como una voz nueva no significaría nada para los demás, tiene que recurrir al repertorio del lenguaje usadero, donde cada voz se encuentra ya adscrita a una significación. A fin de hacerse entender, elige la palabra cuyo usual sentido tenga alguna semejanza con la nueva significación. De esta manera, el término adquiere la nueva significación al través y por medio de la antigua, sin abandonarla. ${ }^{10}$

Es la última frase la que quizá más claramente muestra la diferencia a que me quiero referir, pues considero que, para Kuhn, el investigador no sólo abandona la antigua significación de algunos términos (no de todos) al recalar en un nuevo paradigma, sino que esto es condición necesaria para que la inconmensurabilidad tenga sentido. Aunque de pasada, más abajo ahondo en esta cuestión y sugiero alguna bibliografía ${ }^{11}$; pero creo que es pertinente esta explicitación en el cuerpo del texto.

Debo añadir también que aquí voy a mostrar no sólo el paralelismo entre los dos autores, sino que voy a sugerir que el mismo se produce en base a una común comprensión de la noción de creencia; sin embargo, podrían indicarse muchas otras causas para dicho paralelismo. En especial, ambos autores tienen una deuda (en ambos casos reconocida) con Max

7. OC, III, 562 .

8. Curiosamente, se podría realizar una lectura orteguiana de la biografía de Kuhn: el autor estadounidense habría intentado cumplir su vocación, pues siguió estudiando física, aunque ya no le apasionase, por ayudar en la II Guerra Mundial (cf. Pardo, C. G.: La formación intelectual de Thomas S. Kuhn. Una aproximación biográfica a la teoría del desarrollo científico, Pamplona: Ediciones Universidad de Navarra, 2001, pp. 14-16).

9. $O C$ II, 505 ss.

10. $O C$ II, 506.

11. Cf. infra, nota 31 .

Thémata. Revista de Filosofía No54 (2016) pp.: 53-72. 
Weber; y también, por ejemplo, con Cassirer ${ }^{12}$. Sobre este punto debo indicar algo que no puedo demostrar, pero que me parece plausible: aunque es obvio que Ortega no leyó a Kuhn (pues murió en 1955, y el primer texto del norteamericano que le podría haber interesado, y de hecho le hubiera encantado, La revolución copernicana, es de 1957), no me parece imposible, ni siquiera improbable, que Kuhn sí leyese a Ortega. Éste fue un autor traducido al inglés y muy difundido en Estados Unidos. A este respecto destaca, al igual que en otros ámbitos, la presencia de La rebelión de las masas; sin embargo, quiero llamar la atención sobre el hecho de que Historia como sistema apareció primero en lengua inglesa, en 1935 (época de máxima presencia de Ortega en la filosofía contemporánea), justamente en un libro-homenaje a la obra de Cassirer. No hay prueba alguna, insisto, de que Kuhn leyera al filósofo español, pero éste no era en el ámbito anglosajón ningún desconocido. Más abajo, no obstante, se plantean otras posibles causas de la semejanza entre sus planteamientos, que serán lo que aquí nos preocupará más.

Igualmente, me gustaría destacar otro paralelismo en el que no profundizaré aquí, pero que me parece de magnífica importancia y que espero explotar en otro lugar: para Kuhn, después de una revolución ${ }^{13}$ se produce, de manera más o menos darwinista, una especiación (mejor diríamos una especialización) en las ciencias: donde había una disciplina pasa a haber una ramificación de sub-disciplinas que, con el tiempo, adquirirán su propio peso. Curiosamente, Ortega verá en La rebelión de las masas que esta creciente especialización lleva a un grave problema sociológico: crece el número de individuos muy especializados en un restringido campo de la ciencia (lo cual no es malo de por sí) que, por ser expertos en su terreno, pasan a considerarse expertos en todos los aspectos de la vida humana (lo

12. La influencia de Max Weber en Ortega me parece reclamar un verdadero estudio a fondo: excede los límites que el propio Ortega explicita en "La interpretación bélica de la historia», $O C$ II, 633 ss. Por citar sólo algunos pasajes bien conocidos, se podrían rastrear huellas weberianas en «Notas del vago estío», OC II, 531-565 (especialmente en p. 549) o en España invertebrada (OC III, 441 ss.). Respecto a la presencia de Weber en Kuhn, cf. «Las ciencias naturales y las humanas», en Kuhn, T. S.: El camino desde la estructura, Ensayos filosóficos 1970-1993, con una entrevista autobiográfica, Barcelona: Paidós, 2002, p. 257, donde se explicita también su deuda con Cassirer; o Mayoral de Lucas, J. V.: "Las Conferencias Lowell de Kuhn: un estudio crítico", en Theoria 78, 2013, pp. 459-76.

13. Me permito aquí cierta laxitud, no pormenorizando sobre el concepto kuhniano de revolución científica; sin embargo, no le falta razón a Andrés Rivadulla cuando señala que «Las propiedades características del concepto de revolución científica [en Kuhn] son naturalmente difusas» (Rivadulla, A.: Revoluciones en física, Madrid: Trotta, 2003, p. 14).

Thémata. Revista de Filosofía ํ54 (2016) pp.: 53-72. 
cual sí es grave). A esto lo denominará «la barbarie del especialismo» ${ }^{14}$. Curiosamente, para Kuhn la especialización extrema, si se me permite decirlo así, también es un precio que hay que pagar por tener herramientas cognitivas cada vez más potentes ${ }^{15}$. Sea como fuere, y como ya he adelantado, este aspecto de mi investigación está aún en sus inicios.

Por último, quiero también indicar un paralelismo que habrá de ser investigado entre estos autores: ambos se apoyan en teorías biológicas más o menos comunes para tratar de explicar su postura. Kuhn se preguntará en cierto momento lo siguiente: «iSon las criaturas las que se adaptan al mundo o el mundo en que se adapta a las criaturas? ${ }^{16}$, concluyendo que ambos, nichos y criaturas, evolucionan a la vez. Recuerda esto a las teorías que Ortega considera pertinentes y que le llevan a afirmar, en sintonía con la tesis kuhniana que acabo de señalar, que el medio no es sólo el correlato del cuerpo biológico, sino un órgano del mismo: el de la excitación ${ }^{17}$; lo cual no es difícil vincular con la célebre expresión «Yo soy yo y mi circunstancia» ${ }^{18}$. Y así podríamos seguir largo rato: ambos autores son anti-progresistas en el sentido de que no suponen una teleología en el desarrollo histórico de la ciencia ${ }^{19}$; comparten el papel fundamental de la narración si se trata de atender a la historia ${ }^{20}$; entienden de forma similar la transformación del sujeto de la ciencia (que ha pasado de ser una mente puramente lógica a «un ser humano con sus dimensiones racionales, irracionales y emotivas, -que entran en juego inevitablemente en el manejo de los problemas científicos-... ${ }^{21}$ ), etc. Debo añadir que los pocos estudios serios que hasta ahora se han llevado a cabo para comparar a los dos autores que estoy considerando se han quedado, a mi parecer, muy cortos,

14. Cf. La rebelión de las masas, capítulo XII: «La barbarie del especialismo» (OC IV, 441 ss.). Hay que advertir que esta idea aparece en más ocasiones -se la llame o no por tal nombreen la obra de Ortega (cf. «Prólogo a Historia de la filosofía, de Karl Vorländer», OC III, 401).

15. Cf. Kuhn, T. S.: «El camino desde la estructura», op. cit., pp. 121-122.

16. Kuhn, T. S.: «El camino desde la estructura», op. cit., p. 127.

17. OC III, 730 .

18. Ortega y Gasset, J.: Meditaciones del Quijote, en $O C$ I, p. 757.

19. Sobre el anti-progresismo de Ortega, cf. Conill Sancho, J.: "Más allá del progresismo: la reforma del hombre desde la perspectiva de Ortega", en Anuario Filosófico, 44/2, 2011, pp. 253-275.

20. La razón histórica se entiende, precisamente, en relación con la narración. Muy resumidamente, puede consultarse: De Haro Honrubia, A.: "La antropología social en la obra de Ortega. Su contribución a la etnografía moderna", en Anales del Seminario de Historia de la Filosofía, Vol. 29, Núm. 1, 2012, p. 220; respecto a Kuhn, él mismo lo señala: cf. Kuhn, T. S.: «El problema con la filosofía de la ciencia histórica», en El camino desde la estructura, op. cit., p. 139.

21. Pardo, C. G.: La formación intelectual de Thomas S. Kuhn..., op. cit., p. 12.

Thémata. Revista de Filosofía No54 (2016) pp.: 53-72. 
señalando sólo los parecidos más obvios entre ambas filosofías ${ }^{22}$; o bien se han dedicado a estudios aplicados de conceptos de uno y otro, bien sea para analizar menciones a autores en revistas científicas ${ }^{23} \mathrm{u}$ orientados al estudio del autismo y los surfactantes ${ }^{24}$.

\section{En torno a la historia de la ciencia. Sensibilidad y generaciones}

Una vez hechas las advertencias previas, procede señalar que, para Ortega, la historia en general (y la historia de la ciencia en particular) se comprende como un proceso dinámico, en contra de las posturas adinámicas típicas de los filósofos racionalistas que le antecedieron y que habrían caído «en el error intelectualista que atribuye a la inteligencia una sustantividad e independencia que no tiene» ${ }^{25}$. A su modo de ver, el motor de la historia son las generaciones, esto es, los distintos grupos humanos cuyos miembros son contemporáneos, coetáneos (nacidos en torno a una horquilla de quince años) y con algún tipo de contacto vital ${ }^{26}$. Además de estas características, o precisamente por ellas, se producirá en los individuos que forman parte de una misma generación una similar sensibilidad, aspecto central en este trabajo. Por sensibilidad debemos entender, dicho de manera muy breve, no sólo cierta afinidad por unas u otras corrientes políticas, artísticas o científicas, sino también la consideración común de aquello a lo que se presta atención (consciente o inconscientemente) y

22. Por ejemplo, puede verse Álvarez, J. R.: "Crisis de creencias y revoluciones científicas: Ortega y Kuhn pasado el siglo XX”, en Revista de Estudios Orteguianos, No 6, 2003, pp. 101118. Aunque sólo menciona a Kuhn de pasada, es también muy enriquecedor el texto siguiente: Miquel, M.: "Filosofía de la ciencia en Ortega y Gasset. Ciencias naturales y humanas: hacia una demarcación", en Anales del Seminario de Metafísica, Núm. Extra. Homenaje a S. Rábade, 1992, pp. 127-151 (mención a Kuhn: p. 135). También se ha investigado el uso de la metáfora en los dos autores que estoy considerando: Checa, J. M.: «La metáfora en Ortega y en Kuhn», en Lasaga., L., Navarro, J. M. y San Martín, J. (eds.): Ortega en pasado y en futuro. Medio siglo después, Biblioteca Nueva, Madrid 2007. Comunicación en formato CD-ROM.

23. Cf. Bornmann, L., de Moya Anegón, F. y Leydesdorff, L.: "Do Scientific Advancements Lean on the Shoulders of Giants? A Bibliometric Investigation of the Ortega Hypothesis", en PLoS ONE, October 2010, Volume 5, Issue 10, pp. 1-6.

24. Bailón-Moreno, R. et alii: "The pulsing structure of science: Ortega y Gasset, Saint Matthew, fractality and transfractality", en Scientometrics, Vol. 71, No. 1, 2007, pp. 3-24.

25. OC VI, 369.

26. En este sentido, Ortega admite la posibilidad (para él futura, efectiva hoy) de que en distintos periodos históricos sean parte de una misma generación personas nacidas en distintos lugares: la globalización del tiempo presente permite que cualquier ciudadano europeo entre hoy en contacto con un coetáneo, por ejemplo, japonés, pudiendo formar ambos parte de una misma generación; en la España de 1930, en cambio, este contacto intercultural dificultaba enormemente que los miembros de una misma generación fueran de lugares muy alejados.

Thémata. Revista de Filosofía $\mathrm{N}^{\circ} 54$ (2016) pp.: 53-72. 
aquello que, en cambio, pasa desapercibido. Tal sensibilidad sería, entonces, la que nos diferencia del hombre primitivo, ya que nos hace variar la interpretación que hacemos de unos hechos que son los mismos:

Desde el salvajismo hasta nuestros días no creo que se haya inventado ninguna sensación ni sentimiento elemental: la materia, pues, el conjunto de hechos brutos que nos preocupan es el mismo que preocupaba al salvaje; si en algo nos separamos de él habrá que buscarlo en la distinta valoración que a aquellos mismos motivos o hechos demos. ${ }^{27}$

Y a ello añadirá, en las ya centenarias Meditaciones del Quijote, que «...la nueva sensibilidad suscita nuevos usos e instituciones, nueva arquitectura y nueva poesía, nuevas ciencias y nuevas aspiraciones, nuevos sentimientos y nueva religión». ${ }^{28}$ Fácilmente advertimos aquí que el concepto de sensibilidad que Ortega maneja en relación con las sociedades en general guarda cierto vínculo con el paradigma, esquema conceptual o la matriz disciplinaria ${ }^{29}$ que Kuhn pone en relación con esos grupos, más restringidos, que son las comunidades de científicos: el paradigma ${ }^{30}$ imperante determina lo que los científicos adscritos a él "ven" o "no ven" 31.

Pero volvamos a Ortega. Desde su punto de vista, en cualquier momento conviven (dicho sea de una forma muy general) en un mismo espacio cuatro generaciones: la de los niños (0-15 años de edad), la de los jóvenes

27. $O C \mathrm{I}, 386$.

28. OC I, 792.

29. Kuhn maneja estos tres conceptos para remitirse a la misma idea, intentando superar con unos las críticas o deficiencias de los otros. Así, hablará de paradigma, como es bien conocido, en La estructura de las revoluciones científicas; se referirá al esquema conceptual tanto en La revolución copernicana como en El camino desde la estructura; y empleará la noción de matriz disciplinaria, fundamentalmente, en La tensión esencial y en el Postscriptum de 1969 a La estructura de las revoluciones cientificas.

30. Es bien conocida la controversia que generó el empleo, por parte de Kuhn, del concepto de paradigma; para un rastreo histórico en torno a esta noción, que Kuhn no habría llevado a cabo, cf. Santibáñez Yáñez, C.: "Ciencia, inconmensurabilidad y reglas: crítica a Thomas Kuhn”, en Revista de Filosofía, Volumen 64, 2008, pp. 163-167.

31. Sobre este punto quiero explicitar algo que, en algunas ocasiones, se malinterpreta. Para Kuhn, el científico en tanto que tal no ve una cosa X como A o como B, pudiendo alternar entre ambas "visiones"; no hay para él (para el científico) una autoridad que le diga que X es en realidad A o B (cf. Kuhn, T. S.: La estructura de las revoluciones científicas, México: FCE 2006, capítulo X). Aunque con esto se entra en problemas que exceden el interés de este artículo, debo añadir que en torno a este asunto se han de considerar dos aspectos de la teoría kuhniana que están en relación entre sí: el problema referente a la traducción y la interpretación, por una parte (respecto a lo cual recomiendo la lectura de Fernández Moreno, L.: "La noción de inconmensurabilidad en Kuhn”, en Llull, vol. 18, 1995, pp. 441-456); y la cuestión en torno a la reversibilidad de la conversión desde un paradigma a otro (cf. infra, nota 56).

Thémata. Revista de Filosofía No54 (2016) pp.: 53-72. 
(15-30), la de los hombres y mujeres que pujan por hacerse con el control de las instituciones -políticas, científicas (cátedras), etc.- (30-45 años) y la de los que en el momento ostentan, precisamente, tal control (45-60 años) ${ }^{32}$. De esta manera, son los dos últimos grupos los que realmente marcan el temple de cada momento histórico. Sin embargo, conviene explicitar una tesis de Ortega que resulta fundamental para nuestros actuales intereses. Por cuestión de tiempo vital (esto es: porque la gente incurre irremediablemente en su propio deceso), la generación más madura acabará siendo sustituida por la siguiente, y ésta puede mantener respecto a aquélla dos posturas: beligerante o continuista; pero siempre se mantiene relación de continuidad (aunque sea rupturista) con lo anterior. En palabras de Kuhn, «...no hay ningún esquema conceptual que nazca de la nada» ${ }^{33}$.

A propósito de todo esto, no puedo dejar de mencionar lo siguiente: la tradición es un elemento fundamental, tanto en la concepción de Ortega como en la de Kuhn, para comprender el avance histórico (de la ciencia), aunque sea porque es el "algo" contra el que se rebelan los revoluciona$\operatorname{rios}^{34}$ (sépanlo o no) ${ }^{35}$. Así, puede sucederse un buen número de generaciones en las cuales los cambios de sensibilidad sean mínimos -aunque haya en tal desarrollo avances considerables tanto en ciencia como en diversos ámbitos, fundamentalmente por el avance de la técnica-, alternándose estos periodos de paz con otros en los que las generaciones entrante y saliente permanezcan en actitud combativa (momentos en que aparecen lo que Ortega denomina generaciones decisivas, conocidas por su epónimo ${ }^{36}$ ). Aunque este punto, y su relación con Kuhn, quedará más claro un poco más adelante, podemos ya intuir aquí momentos revolucionarios que alternan históricamente con momentos continuistas (ya se den éstos durante el imperio de una generación o bien en periodos más extensos: cuando sucesivas generaciones no quiebran el paradigma dominante, dicho en términos de Kuhn, o cuando no se producen crisis de creencias fundamentales, dicho

32. La desatención respecto a los mayores de 60 años se debe exclusivamente a que, en la consideración orteguiana, las personas de esa edad van poco a poco desapareciendo de la vida pública, aunque no fallezcan.

33. Kuhn, T. S.: La revolución copernicana, op. cit., p. 71.

34. En este sentido, quiero señalar que la sistematicidad del pensamiento orteguiano al que me referí en la nota 1 resulta decisiva en lo tocante al pensamiento de la revolución. Así, la manera en que comprende la revolución político-social (cf. «Hacia una mejor política», en $O C$ III, 48-49) encaja de manera extraordinariamente precisa tanto con su concepción del desarrollo científico como con la noción de revolución que maneja Kuhn.

35. Sobre el papel de la tradición en el pensamiento de Thomas Kuhn, cf. Tafoya Ledesma, E.: "El valor de la tradición en Thomas Kuhn: continuidad y principio de cambio", en Acta Sociológica, núm. 52, mayo-agosto de 2010, pp. 77-98.

36. Esta idea encaja aceptablemente bien con el «Epílogo» de Kuhn, T. S.: La estructura de las revoluciones científicas, op. cit., p. 311.

Thémata. Revista de Filosofía $\mathrm{N}^{\circ} 54$ (2016) pp.: 53-72. 
en términos de Ortega). Además, destacará el filósofo madrileño que tras producirse un cambio en las creencias imperantes en la época, algunos términos se siguen manejando con significado diferente, rasgo típico de la inconmensurabilidad kuhniana ${ }^{37}$. Esta idea la expresa muy bien Alfred Schütz, en un texto que, dicho sea de paso, poco o nada parece tener que ver con la cuestión (aunque en realidad está manejando ideas de William James con un trasfondo orteguiano, algo que será determinante para nosotros como mostraré después). Así, señala Schütz:

\begin{abstract}
Nuestra relación con el mundo social se basa en el supuesto de que, a pesar de todas las variaciones individuales, el prójimo experimenta los mismos objetos esencialmente de la misma manera que nosotros, y viceversa; y también que sus esquemas de interpretación y los nuestros ofrecen la misma estructura típica de conexiones. Si esta creencia en la identidad esencial de la experiencia intersubjetiva del mundo se viene abajo, entonces se destruye la posibilidad misma de establecer comunicación con el prójimo ${ }^{38}$.
\end{abstract}

37. Lo explica muy bien Juan Vicente Mayoral de Lucas: «Sólo podemos conocer a fondo fases de la historia de la ciencia tras aprender los modos de expresión propios de cada una de esas fases (es decir, si aprendemos su propio lenguaje científico). No hay en Kuhn nada parecido a una "forma general de pensamiento científico", sin los ulteriores matices que hacen de esa expresión algo trivial» (Mayoral de Lucas, J. V.: "Cómo ser un buen historicista: Thomas Kuhn y el Programa HPS de Princeton”, en Daimon. Revista de Filosofía, suplemento 1, 2007, p. 118.

38. Schütz, A.: "Don Quijote y el problema de la realidad", en Diánoia, vol. 1, n 1, 1955, p. 318. No es casualidad que Schütz salga aquí a nuestro encuentro. Así, hablamos de un discípulo de Husserl, autor cuya concepción de las creencias no debemos obviar al abordar el modo en que Ortega maneja dicha noción (en especial, si atendemos a obras como Sistema de la psicología, OC VII, 427-534). Es muy interesante a este respecto la tesis doctoral de Rafael Lorenzo Alquézar El concepto de creencia en Ortega, llevada a cabo en la UNED (Madrid) en 1999, dirigida por Javier San Martín (que se manifiesta en una línea similar al vincular a Ortega con la fenomenología en su conocido texto San Martín, J.: Fenomenología y cultura en Ortega: ensayos de interpretación, Madrid: Tecnos, 1998). Cabe notar que Lorenzo filia la noción orteguiana de creencia (antes de 1923, y especialmente en 1915-1916), como ya he señalado, en la fenomenología husserliana; sin embargo, él mismo deja abierta la puerta -y es parte de lo que yo estoy tratando de poner especialmente de manifiesto- al papel jugado por la obra de William James en dicha apropiación conceptual; cf. Lorenzo Alquézar, R.: "Fenomenología de la creencia. La primera reflexión sobre el concepto de creencia en Ortega y Gasset”, en Studium. Revista de Humanidades, 13, 2007, p. 168; o en la tesis doctoral mencionada, cap. I.2., donde a Husserl y James añade como influencia la obra de Dilthey. Por otra parte, debo indicar que el propio Ortega no duda en poner en relación al pensador germano con el norteamericano (por ejemplo, en "El problema del conocimiento", OC VII, 58, n. 6), aunque la crítica se haya centrado mucho más en la influencia del primero que en la del segundo (aspecto que justifica la "ceguera" a la hora de poner en relación el pensamiento de nuestro autor con la filosofía estadounidense). A todo ello habría que añadir también la presencia del concepto de creencia en la filosofía francesa, particularmente en Le Bon (al que, por cierto, no se refiere Lorenzo en sus, por lo demás, magníficas investigaciones), a la que me referiré brevemente más abajo, etc. La cuestión es excesivamente amplia como para abordarla aquí detenidamente; lo señalado sólo ha de servir de advertencia para no interpretar este texto como una visión simplista y poco documentada sobre el particular.

Thémata. Revista de Filosofía Nº54 (2016) pp.: 53-72. 


\section{Las fuentes históricas del paralelismo}

\subsection{El concepto de creencia}

En lo poco que he dicho hasta ahora apreciará el ojo entrenado una muy similar mecánica en el desarrollo histórico tal y como lo plantean los dos autores que estoy considerando. Sin embargo, quizá sea ahora el momento de buscar, más allá de los paralelismos fácticos, algún elemento teórico que permita insinuar que el paralelismo no es sólo coyuntural, sino también estructural entre ambos.

Para tratar de elucidar este punto, conviene destacar que Ortega opera con una distinción fundamental a lo largo de su filosofía, aunque fuese variando la terminología empleada. Tal distinción es la que tiene lugar entre ideas y creencias: dirá en muchas ocasiones que las ideas (llamadas a veces ocurrencias) las tenemos, mientras en las creencias estamos ${ }^{39}$. El siguiente cuadro, ofrecido por José Lasaga Medina ${ }^{40}$, ofrece una rápida comparativa entre ambos conceptos:

\begin{tabular}{|l|l|}
\hline \multicolumn{1}{|c|}{ CREENCIAS } & \multicolumn{1}{c|}{ IDEAS } \\
\hline «Contar con» & «Reparar en» \\
\hline Carácter de realidad & Carácter revocable: fantasías \\
\hline Continente de la vida humana & Contenidos del yo-conciencia \\
\hline Latentes (o inconscientes) & Patentes a la conciencia \\
\hline Tienen vigencia social & Sin vigencia \\
\hline Son colectivas & Son individuales \\
\hline Son heredadas & Son creadas \\
\hline
\end{tabular}

Lo que más nos puede interesar sobre esta distinción es que las creencias son todo aquello que de uno u otro modo, sin tener conciencia de ello (algo que comparte Kuhn: según él, Brahe no es consciente de su copernicanismo ${ }^{41}$ ), suponemos: que al acabar una clase el pasillo seguirá al otro lado de la puerta del aula; que las leyes de la naturaleza seguirán funcionando mañana, sean las que sean; etc. En el momento en que dejamos

39. Cf. el célebre texto Ideas y creencias (OC V, 659-686).

40. Lasaga Medina, J.: José Ortega y Gasset (1883-1955). Vida y filosofía, Madrid: Biblioteca Nueva - Fundación José Ortega y Gasset, 2003, p. 148.

41. Cf. Kuhn, T. S.: La revolución copernicana, op. cit., p. 268.

Thémata. Revista de Filosofía $\mathrm{N}^{\circ} 54$ (2016) pp.: 53-72. 
de estar en una creencia (en rigor, estamos en un conjunto de creencias que no entrañan incompatibilidad entre sí), esto es, cuando dudamos, la creencia se hace algo consciente, volviendo a ser una idea. Digo que "vuelve" a ser una idea porque también es una idea el origen de la creencia: alguien tiene una ocurrencia inicialmente, pero si sigue siendo efectiva y se transmite de generación en generación acaba siendo algo supuesto o consabido; aún mejor, con una expresión clarísima del propio Ortega, pasa a ser algo con lo que contamos.

Todo este rodeo en torno a las creencias tiene sentido para nuestro tema en la medida en que en todo momento histórico hay una serie de creencias operativas -operan, ante todo, determinando la sensibilidad de la época a que antes me referít ${ }^{42}(y$, en este sentido, son las que hacen de los hombres y mujeres seres históricos ${ }^{43}$ ) - en las distintas colectividades. Entiéndase bien: hay creencias que son compartidas por varias comunidades y otras que sólo afectan a uno u otro grupo. El objetivo de la historia en general como disciplina, y esto incluye a la historia de la ciencia, será estudiar las creencias fundamentales que han estado operando en cada momento de las diversas civilizaciones, señalando las crisis en dichas creencias -esto es, cuando se deja de tener fe viva en ellas y se está en un inestable «mar de dudas», no sabiendo uno a qué atenerse- como los momentos en que dichas civilizaciones o mundos se vienen abajo. Esto, de por sí, puede emparentarse sin demasiada dificultad con el pensamiento kuhniano. Para empezar, el propio Kuhn señala lo siguiente: «...los historiadores siempre captan un proceso ya en marcha, cuyos inicios se pierden en tiempos anteriores. Las creencias ya están ahí: proporcionan la base para la continua investigación cuyos resultados en algunos casos las cambiarán» ${ }^{44}$. En cierto grado, restringido (insisto) al ámbito de las comunidades científicas, los paradigmas juegan un papel similar a las creencias $^{45}$ (según Carlos Gustavo Pardo, para Kuhn «Los paradigmas, o las

42. Sobre la relación entre época y sensibilidad, resulta muy aclarador el siguiente pasaje de una de las obras más brillantes del joven Ortega, "Adán en el paraíso», incluida en Personas, obras, cosas. La cita es la que sigue: «Imaginaos delante de una obra a la moda. Sus figuras incitan nuestra fantasía al movimiento, nos conmueven, viven para nosotros. Pasan cincuenta años y aquellas figuras, ante las pupilas de nuestros hijos, permanecen mudas, quietas, muertas. ¿Por qué han muerto ahora? ¿De qué vivían antes? De nosotros, de nuestra sentimentalidad momentánea, periférica, pasajera» (OC II, 75).

43. Ovejero, A.: "Utilidad de Ortega y Gasset para la actual psicología social”, en Revista de Psicología Social, 2005, 20 (1), p. 8.

44. Kuhn, T. S.: «El camino desde la estructura», en El camino..., op. cit., p. 119.

45. Particularmente, en ciertos pasajes de La estructura notoriamente ambiguos. En textos posteriores en que se vinculan más restringidamente los paradigmas a los ejemplares (por ejemplo, en el «Epílogo» de 1969 a La estructura de las revoluciones científicas, op. cit., p. $320)$, esta relación paradigmas-creencias se ve debilitada.

Thémata. Revista de Filosofía No54 (2016) pp.: 53-72. 
estructuras léxicas, poseen una existencia exclusivamente comunitaria» ${ }^{46}$, lo que podemos relacionar con la caracterización de las creencias orteguianas que he ofrecido), y entran en crisis de similar modo. Pero no perdamos el rumbo: este es otro paralelismo, y ahora estamos buscando supuestos teóricos en Kuhn cuyas consecuencias hayan llevado a que su postura sea similar a la de Ortega.

\subsection{El origen del concepto de creencia: en torno a William James y Alexander Bain}

No está del todo claro de dónde extrae Ortega este concepto de creencia, aunque resulta muy plausible que sea una idea francesa: por ejemplo, Gustave Le Bon la maneja en Psicología de las masas. Un poco más abajo lanzaré otra sugerencia.

¿Hay algún concepto que pueda estar en la base del pensamiento kuhniano similar a este de las creencias? Si retrocedemos al siglo XIX, encontraremos a un autor británico poco conocido en España pero que resultó determinante para el Club Metafísico de Cambridge, esto es, el pequeño grupo de amigos y contertulios en el que nació la corriente pragmatista y del que formaban parte Charles S. Peirce y William James, entre otros. Ese autor es Alexander Bain, y nos interesa advertir que manejó una noción de creencia (belief) tal que la siguiente: «aquello en virtud de lo cual un hombre está dispuesto a obrar» ${ }^{47}$. Desde luego, esta definición de creencia es compatible, incluso complementaria, respecto al concepto que maneja Ortega $^{48}$ (sería en clave individual lo que las creencias orteguianas son

46. Pardo, C. G.: La formación intelectual de Thomas S. Kuhn..., op. cit., p. 75.

47. «...that upon wich a man is prepared to act»: así la resume Charles Sanders Peirce en sus Collected Papers, 5.12; la traducción la he tomado de Sini, C.: El pragmatismo, Tres Cantos: Akal, 1999, p. 15, aunque él no ofrece la referencia de la obra de Peirce. Además, cf. Bain, A. [en línea]: «La creencia», (trad. Armando Daniel Otamendi), extraído de Bain, A.: Mental and Moral Science. Part Frist: Psychology and History of Philosophy, Londres: Longmans, Green and Co, 1868, Book IV: "The Will", Chapter VIII: "Belief", pp. 371-385) <http://www.unav.es/ gep/AlexanderBainLaCreencia.pdf $>$ [Consulta: 5 de junio de 2015].

48. Para Pedro Laín Entralgo es, de hecho, el principal vínculo y semejanza entre Ortega y James. Así, indica: «Una tesis común une a W. James con Ortega y Marías: las ideas acerca de las cosas nos muestran -no siempre- la verdad de ellas, pero son las creencias y sólo ellas las que nos dan certidumbre de la realidad. Sólo cuando no hay discrepancia grave entre las ideas y las creencias llega a ser en alguna medida armoniosa y segura la vida humana» (Laín Entralgo, P.: Ser y conducta del hombre, Madrid: Espasa Calpe, 1996, pp. 328-329).

Thémata. Revista de Filosofía No54 (2016) pp.: 53-72. 
respecto a lo colectivo), permitiendo que autores que basen de uno u otro modo su doctrina en tal concepto lleguen a posturas similares.

No se conocen pruebas de que Kuhn leyese a Alexander Bain; sin embargo, no me parece difícil que la idea de fondo llegase a él de manera indirecta. Así, es bien conocido el papel determinante que el concepto de creencia del autor escocés jugó en el desarrollo posterior del pragmatismo americano, tanto en Peirce como en James ${ }^{49}$; y a estos autores sí los leyó Kuhn. Particularmente importante sería realizar, si aún no se ha llevado a cabo, un análisis de la presencia de La voluntad de creer y otros ensayos de filosofía popular, de James, en la obra kuhniana; libro en el que, por cierto, Bain aparece citado explícitamente de un modo que ha de resultar sorprendentemente familiar para los lectores de Kuhn ${ }^{50}$. Naturalmente, aunque no puedo entrar aquí a fondo en ello, hay claves básicas del pragmatismo norteamericano en torno a las creencias que Kuhn ha asimilado (si es que no lo ha hecho, como he sugerido, leyendo al propio Ortega); por ejemplo, la transitoriedad de dichas creencias aparece mencionada muy claramente en La revolución copernican ${ }^{51}$.

En este momento debo añadir algo que antes prometí. He indicado que el concepto de creencia podría haber llegado a Ortega desde la filosofía francesa, y me parece lo más plausible; sin embargo, decía que cabe otra posibilidad, que ahora explicito: que la fuente originaria sea, precisamente, William James. Nuevamente, esto es sólo una hipótesis germinal, pero que me parece interesante señalar: la herencia jamesiana en Ortega, aunque de manera subterránea, es reconocible en la filosofía del pensador español, que incluso se permite aludir, en "Aurora de la razón histórica», a una cierta «voluntad de creer»" ${ }^{52}$. Y podemos ir más allá. Me parece improbable que Ortega leyese directamente la obra de Alexander Bain; sin embargo, e incluso si admitiésemos una lectura sumamente despreocupada de La voluntad de creer y otros ensayos de filosofía popular (que no lo admitimos), resulta que Bain aparece citado en una obra cuya lectura realizó nuestro autor sin ningún género de duda: el Ensayo sobre los datos

49. Sobre este particular es ya un clásico Fisch, M. H.: "Alexander Bain and the Genealogy of Pragmatism", en Journal of the History of Ideas, Vol. 15, No. 3, Jun., 1954, pp. 413-444.

50. Cf. James, W.: The Will to Believe and Other Essays in Popular Philosophy, Cambridge, Massachusetts and London, England: Harvard University Press 1979, pp. 62-63, 76-77, 102, 142 y 263-264. Por otra parte, debo dejar constancia -aunque el asunto es bien conocido y en este trabajo se da por supuesto- de que los planteamientos de Kuhn y sus posibles lecturas de los autores aquí propuestos se vieron en buena medida mediados por la obra de Koyré, aspecto bien conocido y trabajado en la historia de la ciencia y sobre el que existe abundante bibliografía.

51. Cf. Kuhn, T. S.: La revolución copernicana, op. cit., p. 26.

52. $O C \mathrm{~V}, 373$.

Thémata. Revista de Filosofía ํ54 (2016) pp.: 53-72. 
inmediatos de la conciencia, de Bergson ${ }^{53}$ (autor, a su vez, que conoció bien la obra de James, especialmente la de carácter psicológico). Quede aquí el asunto por el momento, pero no se dude de su pertinencia.

\section{La elección de teoría: el papel de los valores}

No me gustaría dejar de señalar, también de manera somera, que algunas de las críticas que Kuhn recibió por La estructura de las revoluciones científicas no sólo podrían extenderse a Ortega, sino que éste hubiera dado respuestas similares a las del antiguo físico. Así, me gustaría destacar en este acelerado acercamiento a la cuestión, la crítica de Lakatos en la que considera que el sistema propuesto por Kuhn estaría conformado particularmente por elementos subjetivos en lo referido a la elección de teorías ${ }^{54}$. La respuesta de Kuhn es conocida en el ámbito de la filosofía de la ciencia ${ }^{55}$, por lo que me gustaría indicar que Ortega daría indicaciones muy similares, empleando para ello conceptos que ya hemos ido mostrando aquí en buena medida: si bien para pasar de un conjunto de creencias a otro ha de tener lugar algo así como una conversión ${ }^{56}$ (de nuevo, similitud con Kuhn, hasta el punto de que la conversión es instantánea, pero en base a buenas razones no definitivas per $s e^{57}$ ), no cabe aquí hablar de mero capricho o subjetividad en el sentido desde el que se criticó al pensador estadounidense. Así, las creencias de la generación a que uno pertenece, como se indicaba en el cuadro facilitado más arriba, tienen carácter de realidad;

53. Cf. Bergson, H.: Ensayo sobre los datos inmediatos de la conciencia, Salamanca: Sígueme, 2006, p. 27.

54. Para un resumen conciso y, no obstante, claro sobre este particular, cf. Rivadulla, A.: Filosofía actual de la ciencia, Madrid: Editora Nacional, 1984, pp. 233-239.

55. El texto central en este sentido es el artículo «Consideraciones en torno a mis críticos», que se encuentra traducido en El camino desde la estructura..., op. cit., pp. 151-209.

56. Mientras exponía públicamente una versión previa y aún muy poco definida de este trabajo, un oyente anónimo me sugirió, con pleno acierto, la pertinencia de relacionar el concepto kuhniano de conversión (y, por tanto, el orteguiano) con el uso que hace del mismo precisamente William James en Las variedades de la experiencia religiosa. En la actualidad estoy trabajando sobre ello, y espero poder dar en próximas fechas a la prensa algunas conclusiones al respecto. Quede en todo caso patente mi agradecimiento al citado oyente anónimo.

57. Quizá sea un buen momento para advertir que, según algunos intérpretes, la conversión que el propio Kuhn experimentó leyendo a Aristóteles, y a la que se refiere en varias ocasiones como ejemplo de a qué se refiere, no fue en absoluto instantánea, sino que vino mediada por los textos de Koyré. Cf. Artetxe, A.: “Qué estaba preparado para ver Thomas Kuhn?”, en Llull, vol. 13, 1990, p. 24. Respecto al uso técnico del concepto de conversión por parte de Kuhn, entre otras fuentes, cf. Kuhn, T. S.: La estructura de las revoluciones cientificas, op. cit., pp. 82-83 y 267-269.

Thémata. Revista de Filosofía No54 (2016) pp.: 53-72. 
dicho de otro modo, el individuo (por ejemplo, en tanto que científico ${ }^{58}$ ) ha de responder a una serie de exigencias de su tiempo que en modo alguno son arbitrariedades derivadas de apetencias individuales.

Profundizando un poco más, debo señalar que Kuhn y Ortega dotan a los valores de características similares. Para empezar, Ortega considerará que es característica esencial de los valores el ser estimados en aquellos hechos y objetos que son susceptibles de hospedar dichos valores ${ }^{59}$. Tal concepción la encontramos de manera muy similar en el prólogo que Conant escribió a La revolución copernicana, donde afirma explícitamente lo siguiente, cuando compara ciencia y literatura:

...el teatro de Shakespeare ha sido y sigue siendo el objeto de interminables debates en los que se ha criticado desde todo ángulo concebible el estilo y los personajes y constantemente han llegado hasta nosotros palabras de admiración o censura para los mismos. Por otro lado, nadie admira o desaprueba los metales o el comportamiento de sus sales. ${ }^{60}$

Antes de continuar, debo explicitar algo anecdótico pero iluminador: aunque es pura casualidad (dada la universalidad del autor de Hamlet), es precisamente a Shakespeare a quien recurre Ortega a la hora de condensar uno de los puntos tratados en este artículo. Así, dirá en «Renan» que «En el siglo XVIII un francés no lograba asimilarse la pasión acre de Shakespeare; hoy podemos sentir el corazón de Julieta estremeciéndose en el cuerpo de porcelana de una japonesa» ${ }^{61}$.

Retomando la argumentación y la cita de Conant, vemos que a la hora de elegir entre diversas teorías, Kuhn señala que entran en juego diferentes valores (coherencia, amplitud, precisión, simplicidad y fecundidad) que ayudan a optar por una u otra de entre las teorías paradigmáticas posibles -aunque, y este es un cierto relativismo que Kuhn encuentra aceptable, cada época o individuo tiende a dotar de mayor o menor peso a uno u otro valor. Respecto a Ortega, considerará que los valores son objetivos, y también es objetiva la jerarquía entre ellos; pero la objetividad viene dada por el contexto, hasta el punto de señalar que los valores se

58. Aunque hasta aquí ha sido un supuesto de este artículo, debo aclarar que, para Kuhn, los científicos tienen también otras "caras": además de ejercer sus investigaciones, tienen creencias religiosas o metafísicas, circunstancias personales, etc. (lo cual concuerda muy bien con la filosofía orteguiana). Cf. La estructura de las revoluciones científicas, op. cit., pp. 80 y 271.

59. OC III, 544.

60. En Kuhn, T. S.: La revolución copernicana, op. cit., p. 17.

61.OC II, 45 .

Thémata. Revista de Filosofía No54 (2016) pp.: 53-72. 
descubren o crean históricamente ${ }^{62}$. Si a ello añadimos que considerará a los valores como ideales orientadores de la acción ${ }^{63}$ (en todos los ámbitos y, por ende, en la ciencia), las decisiones entre teorías o paradigmas no son cuestiones meramente subjetivas.

\section{Conclusión}

A pesar del inevitable atropellamiento, creo que el presente artículo prueba, en primer lugar, que tiene pleno sentido empezar a realizar investigaciones desde un punto de vista orteguiano en torno a la historia de la ciencia, pues si a Kuhn se le ha concedido (merecidamente) un papel fundamental en tal disciplina, y en la filosofía de la ciencia en general, tanto más habrá que otorgar a quien le antecedió y tuvo con el norteamericano tantos puntos en común. Igualmente, espero haber abierto, a través de lo que hoy son meras sugerencias e hipótesis, una nueva vía para el estudio de la influencia de pensadores británicos y estadounidenses en la obra de Ortega y Gasset, y la posible presencia de éste en aquéllos. Del mismo modo, y en último lugar, la convergencia entre el pensamiento de Kuhn y el del pensador madrileño habilitan la posibilidad de realizar, en un futuro no tan lejano, lecturas pragmatistas de la obra orteguiana, dados los comunes cimientos entre ambas corrientes, permitiendo quizá la superación de obstáculos hoy infranqueables para tal filosofía.

\section{Referencias bibliográficas}

Álvarez, J. R.: "Crisis de creencias y revoluciones científicas: Ortega y Kuhn pasado el siglo XX”, en Revista de Estudios Orteguianos 6, 2003, pp. 101-18.

Artetxe, A.: “¿Qué estaba preparado para ver Thomas Kuhn?”, en Llull, 13, 1990, pp. 21-42.

Bailón-Moreno, R., et al.: "The Pulsing Structure of Science: Ortega y Gasset, Saint Matthew, Fractality and Transfractality", en Scientometrics, vol. 71, $\mathrm{n}^{0}$ 1, 2007, pp. 3-24.

Bain, A. [en línea]: «La creencia», (trad. Armando Daniel Otamen-

62. OC III, 549 .

63. $O C$ II, 278.

Thémata. Revista de Filosofía №54 (2016) pp.: 53-72. 
di), extraído de Bain, A.: Mental and Moral Science. Part Frist: Psychology and History of Philosophy, Londres: Longmans, Green and Co, 1868, Book IV: "The Will", Chapter VIII: "Belief", pp. 371-385) <http://www.unav.es/ gep/AlexanderBainLaCreencia.pdf $>$ [Consulta: 5 de junio de 2015].

Bornmann, L., de Moya Anegón, F. y Leydesdorff, L.: "Do Scientific Advancements Lean on the Shoulders of Giants? A Bibliometric Investigation of the Ortega Hypothesis", en PLoS ONE, October 2010, Volume 5, Issue 10, pp. 1-6.

Brunetti, J.: "Thomas Kuhn: ¿epistemólogo o psicólogo de la ciencia?", en Revista CTS, no 22, vol. 8, Enero de 2013, pp. 191-212.

Checa, J. M.: «La Metáfora En Ortega Y En Kuhn», en Lasaga, J., Navarro, J. M., y San Martín, J. (eds.): Ortega En Pasado Y En Futuro. Medio Siglo Después, Madrid: Biblioteca Nueva 2007, CD-ROM.

Conill Sancho, J.: "Más allá del progresismo: la reforma del hombre desde la perspectiva e Ortega", en Anuario Filosófico 44/2, 2011, pp. 253-275.

De Haro Honrubia, A.: "La antropología social en la obra de Ortega. Su contribución a la etnografía moderna", en Anales del Seminario de Historia de la Filosofía, vol. 29, n ${ }^{\circ}$ 1, 2012, pp. 217-40.

Fernández Moreno, L.: "La noción de inconmensurabilidad en Kuhn”, en Llull 18, 1995, pp. 441-456.

Ginev, D.: "Rereading Normal Science", en CRÍTICAS. Revista Hispanoamericana de Filosofía, vol. 35, no 105, 2003, pp. 65-89.

James, W.: The Will to Believe and Other Essays in Popular Philosophy. Cambridge, Massachusetts and London, England: Harvard University Press, 1979.

Kuhn, T. S. The Copernican Revolution : Planetary Astronomy in the Development of Western Thought. Cambridge, Mass; London: Harvard University Press, 1957.

--- La revolución copernicana. La astronomía planetaria en el desarrollo del pensamiento. Barcelona: Ariel, 1996.

--- El camino desde la estructura. Ensayos filosóficos 1970-1993, con una entrevista autobiográfica. Barcelona: Paidós, 2002.

--- La estructura de las revoluciones científicas. México: FCE, 2006.

--- The Structure of Scientific Revolutions. 3 ed. Chicago and London: The University of Chicago Press, 1996.

--- La tensión esencial: Estudios selectos sobre la tradición y el cambio en el ámbito de la ciencia. Madrid: FCE, 1993.

Kuukkanen, J.-M.: "Rereading Kuhn", en International Studies in the Philosophy of Science vol. 23, n ${ }^{\circ}$ 2, 2009, pp. 217-224.

Lasaga Medina, J.: José Ortega Y Gasset (1883-1955). Vida y filosofía. Madrid: Biblioteca Nueva - Fundación José Ortega y Gasset, 2003. 
Laín Entralgo, P.: Ser y conducta del hombre. Madrid: Espasa Calpe, 1996.

Lorenzo Alquézar, R.: "Fenomenología de la creencia. La primera reflexión sobre el concepto de creencia en Ortega y Gasset", en Studium. Revista de Humanidades, 13, 2007, pp. 141-170.

--- El concepto de creencia en Ortega (Tesis doctoral), Madrid: UNED, 1999. Dirigida por Dr. Javier San Martín.

Mayoral de Lucas, J. V.: "Cómo ser un buen historicista: Thomas Kuhn y el programa HPS de Princeton", en Daimon. Revista de Filosofía, suplemento 1, 2007, pp. 113-119.

--- "Las Conferencias Lowell de Kuhn: un estudio crítico", en Theoria 78, 2013, pp. 459-76.

Miquel, M.: "Filosofía de la ciencia en Ortega y Gasset. Ciencias naturales Y humanas: hacia una demarcación”, en Anales del Seminario de Metafísica, Núm. Extra. Homenaje a Sergio Rábade, 1992, pp. 127-151.

Ortega y Gasset, J.: Obras Completas. 10 vols. Madrid: Taurus Fundación José Ortega y Gasset, 2004-2010.

Ovejero, A.: "Utilidad de Ortega y Gasset para la actual psicología social”, en Revista de Psicología Social 20 (1), 2005, pp. 3-17.

Pardo, C. G.: La formación intelectual de Thomas S. Kuhn. Una aproximación biográfica a la teoría del desarrollo científico. Pamplona: Ediciones Universidad de Navarra, 2001.

Rivadulla, A.: "Metáforas y modelos en ciencia y filosofía", en Revista de Filosofía, vol. 31, n 2, 2006, pp. 189-202.

--- Revoluciones en física. Madrid: Trotta, 2003.

-- Filosofía actual de la ciencia. Madrid: Editora Nacional, 1984.

--- "Inconmensurabilidad y relatividad. Una revisión de la tesis de Thomas Kuhn", en Revista de Filosofía, vol. 28, n 2, 2003, pp. 237-259.

San Martín, J.: Fenomenología y cultura en Ortega: ensayos de interpretación, Madrid: Tecnos, 1998.

Sánchez Ron, J. M.: "Einstein y la filosofía del siglo XX", en Arbor. Ciencia, pensamiento y cultura CLXXXIII 728, 2007, pp. 833-853.

Santibáñez Yáñez, C.: "Ciencia, inconmensurabilidad y reglas: crítica a Thomas Kuhn", en Revista de Filosofía 64, 2008, pp. 163-182.

Schütz, A.: "Don Quijote y el problema de la realidad", en Diánoia, vol. 1, n 1 1, 1955, pp. 312-330.

Tafoya Ledesma, E.: "El valor de la tradición en Thomas Kuhn: continuidad y principio de cambio", en Acta Sociológica 52, mayo-agosto de 2010, pp. 77-98.

Williams, G.: "Ortega Reading Dilthey, and Ideas of Life", en Journal of Spanish Cultural Studies, vol. 5, n 2, 2004, pp. 201-211. 
